\title{
Review of: "Endogenous controls and microRNA profile in female patients with obstructive sleep apnea"
}

\author{
ANNAROSA ARCANGELI ${ }^{1}$
}

1 University of Florence

Potential competing interests: The author(s) declared that no potential competing interests exist.

\section{Post-Publication Review comments}

Journal: Scientific Reports

Manuscript ID: https://doi.org/10.1038/s41598-022-05782-y

Manuscript Type: Original Research Article

Date invited: $7^{\text {th }}-$ Feb-2022

Title: Endogenous controls and microRNA profile in female patients with obstructive sleep apnea The Authors present a paper entitled “Endogenous controls and microRNA profile in female patients with obstructive sleep apnea".

According to prevous studies by this group of authors, circulating microRNAs represent valuable biomarkers for characterizing obstructive sleep apnea (OSA) in males. The main aim of this study is to identify a set of miRNAs acting as endogenous controls (normalizers for RT-qPCR relative quantification) in female patients with OSA. Then, to analyze differences in the miRNA expression profile between patients with and without OSA. The profiling of 188 miRNAs consistent in plasma samples was performed in a discovery cohort (21 patients) using TaqMan-Low-Density-Array. The ECs were identified by mean centre + standard deviation normalization and concordance correlation restricted normalization. Differentially expressed candidate miRNAs were selected for RT-qPCR validation in a larger and independent validation cohort (consisting of 64 patients). Three circulating miRNAs (miR-30a-5p, miR-93-3p and miR-532-5p) were identified as the best candidates for use as ECs. Twenty-seven miRNA candidates were identified as differentially expressed between OSA and non-OSA patients in the validation cohort but showed no statistically significant differences in the circulating miRNA profile in female patients with and without OSA. The topic is of extreme relevance and of large interest to these days. The problem is well presented, and the background sufficiently described in the Introduction. The study is really well done, based on wellestablished methods and evidences, and the conclusions are reasonable. The criteria used for patients' inclusion/exclusion are clear and well described in the method section as well as in the flowchart of the study.Despite not resulting in any relevant candidate miRNA-based biomarker for obstructive sleep apnea (OSA) in female patients, the present paper provides endogenous controls (ECs), which might represent a relevant factor for future studies on circulating miRNAs (ci-miRNAs) in female patients with OSA. The 
selection of cohort- and disease-specific endogenous normalizers for ci-miRNAs quantification by RT-qPCR is indeed suggested and represents the most accurate approach for standardization in ci-miRNAs relative quantification, considering that widely accepted normalizers for circulating miRNAs relative quantification are still not available (on the contrary of miRNAs derived for cellular/tissue samples). The methodology applied for endogenous candidate EC selection is indeed valid and includes all the currently accepted statistical approaches and bioinformatic tools. Moreover, the fact that candidate EC selection was performed sequentially in a screening cohort followed by a larger and independent validation cohort makes the obtained results highly relevant.

\section{COMMENTS:}

- The authors could have mentioned if any sample size calculation was performed.

- The authors could have declared why PFP plasma was not preferred, considering the potential confounding role of aspecific miRNAs release from platelets

- A statement regarding the absence of any sign of samples hemolysis (whether assessed macroscopically or using molecular approaches) could also have been included in the relative method section.

- If possible, it would have been interesting if an overall ranking of the candidate endogenous controls resulting from the validation phase was provided.

- Even if no differentially expressed miRNA between OSA and non-OSA study groups emerged from the validation phase of the study using the best identified ECs, it would be interesting if any deregulated miRNA entity emerged from subsets of OSA patients presenting with specific clinical characteristics (in terms of demographic and clinical variables, sleep parameters, medical history but also prognosis) when compared to healthy controls. This could highlight potential discriminatory ability of single miRNAs or multiple miRNAs combinations in clinically-defined patients subsets (single marker ROC curve analysis, combined ROC curve analysis).

- Any correlation of miRNAs included in the validation phase (as the ones emerging as differentially expressed in the discovery phase) with relevant clinical parameters values could also be investigated. 